\title{
Trabajo y cohesión social en el ámbito urbano : tensiones y desafíos en la sociedad de mercado chilena
}

Travail et la cohésion sociale dans l'espace urbain: défis et tensions dans la société de marché chilien

Work and social cohesion in the urban space: tensions and challenges in Chilean market society

\section{Guillermo Wormald y Maria Paz Trebilcock}

\section{OpenEdition}

\section{Journals}

Edición electrónica

URL: https://journals.openedition.org/ideas/982

DOI: $10.4000 /$ ideas.982

ISSN: 1950-5701

Editor

Institut des Amériques

\section{Referencia electrónica}

Guillermo Wormald y Maria Paz Trebilcock, «Trabajo y cohesión social en el ámbito urbano : tensiones y desafíos en la sociedad de mercado chilena», IdeAs [En línea], 5 | 2015, Publicado el 02 junio 2015, consultado el 18 octubre 2022. URL: http://journals.openedition.org/ideas/982 ; DOI: https://doi.org/ 10.4000/ideas.982

Este documento fue generado automáticamente el 18 octubre 2022.

\section{cc)}

Creative Commons - Atribución-NoComercial-SinDerivadas 4.0 Internacional - CC BY-NC-ND 4.0 https://creativecommons.org/licenses/by-nc-nd/4.0/ 


\section{Trabajo y cohesión social en el ámbito urbano : tensiones y desafíos en la sociedad de mercado chilena}

Travail et la cohésion sociale dans l'espace urbain : défis et tensions dans la société de marché chilien

Work and social cohesion in the urban space : tensions and challenges in Chilean market society

Guillermo Wormald y Maria Paz Trebilcock

\section{NOTA DEL AUTOR}

Este trabajo está elaborado en base a los desarrollos del proyecto de investigación, "Cultura de Integración y cohesión social en las ciudades chilenas »(SOC-24) financiado por la Comisión nacional de ciencias y tecnología (CONICYT).Esta investigación se pregunta por las posibilidades para el fortalecimiento de la cohesión social ciudadana que ofrecen : las actuales formas de integración y convivencia en los distintos espacios urbanos públicos e institucionales ; las disposiciones de las personas hacia un mayor nivel potencial de contacto social y ; los fundamentos y valoraciones culturales de las oportunidades existentes de contacto social inter clases. Esta indagación la hemos centrado en algunos ámbitos relevantes de convivencia urbana, tales como : el barrio, la escuela, el mall, los espacios públicos de recreación y el trabajo. Ellos representan espacios centrales de contacto e interacción social, así como también de integración a las oportunidades de vida y bienestar social que brinda la sociedad. 


\section{Introducción}

La sociedad chilena ha sufrido un cambio significativo en las cuatro últimas décadas. A los cambios en el modelo de acumulación, se suma un cambio en las reglas del juego que articulan la vida social y una transformación en sus valores sociales (Wormald G., Brieba D., 2012). Esta transformación tiene que ver con el desarrollo de una nueva sociedad de mercado. Esto es, una sociedad en la cual el mercado aparece como sumamente importante. Por lo tanto, asume un rol clave en la generación de puestos de trabajo, las formas de integración a las oportunidades de vida y de cohesión de los trabajadores.

2 En el caso de la generación de puestos de trabajo e integración a las oportunidades de vida, la pregunta relevante es hasta dónde las nuevas condiciones de acumulación han generado oportunidades de empleo capaces de integrar a los trabajadores al ingreso y la protección social. En otras palabras, hasta donde la expansión de la nueva sociedad de mercado ha impulsado un proceso de expansión en el acceso a la ciudadanía social de los trabajadores.

Por otra parte, en cuanto a la organización del mercado de trabajo la pregunta es hasta dónde las nuevas condiciones de acumulación han generado nuevas formas de flexibilización del trabajo y como ellas han impactado en la posibilidad de generar vínculos al interior del trabajo. Más específicamente, cómo estas nuevas condiciones afectan la experiencia de sociabilidad y asociatividad de los trabajadores urbanos.

Por último, en términos de valoración del « otro » la expansión de la nueva sociedad de mercado parece generar tendencias encontradas. Por una parte, difunde valores asociados al a la competencia, emprendimiento y al esfuerzo individual, y por la otra, introduce un reconocimiento del otro precisamente en función de su naturaleza de trabajador esforzado. La primera de estas tendencias parece estar mejor graficada en datos pre-existentes, en tanto que la segunda, será materia de análisis en este trabajo.

Todos estos antecedentes dan cuenta de un fenómeno de individualización de la vida social, especialmente fuerte entre los jóvenes que son los que han sido formados en la nueva sociedad de mercado. Este fenómeno ha ido acompañado por altas expectativas de movilidad social. Nuevamente, la encuesta Eco-social ${ }^{1}$, señala que casi el $70 \%$ de la población considera que ascenderá socialmente en los próximos 10 años y más del $80 \%$ espera que los hijos mejoren su posición social. Es interesante señalar, que los pobres son más optimistas acerca de sus oportunidades de movilidad social en comparación con la percepción que tiene la población en general de esas oportunidades. Asimismo, un $40 \%$ declara haber ascendido en la escala social en los últimos 10 años vis-a-vis un $30 \%$ que declara haber descendido.

De esta forma, se observa que la sociedad chilena se mueve entre una valorización del esfuerzo individual y una necesidad altamente valorada de una mayor igualdad que hace particularmente relevante la pregunta por los patrones de cohesión social efectivamente existentes en la sociedad.

Genéricamente podemos entender por cohesión social « la fuerza que mantiene unidos a los individuos a una sociedad de la que se sienten parte " (Tironi E., Tironi M., 2008). Como bien lo han señalado estos mismos autores, el problema así definido apela a un conjunto de dimensiones simbólicas, políticas y de experiencia de vida en los espacios en los que las personas desarrollan su vida social. Por otra parte, otros trabajos hacen 
ver que éste es un problema que se relaciona con dos dimensiones fundamentales : una de equidad social que apela a la capacidad de la sociedad para producir una distribución equitativa del poder y del bienestar mediante arreglos institucionales específicamente diseñados para ellos y, la otra, relacionada con el fortalecimiento de la sociedad civil y que refiere a la capacidad que ella tiene para generar redes de cooperación y vínculos entre extraños. (Valenzuela E., 2008).

8 Estas formulaciones dejan entrever que la cohesión social es un fenómeno complejo, de aristas diversas y que trascienden el papel que puede jugar el trabajo como fundamento de ella. Sin embargo, indirectamente, ellas también relevan la importancia que la organización y valoración del trabajo puede tener para robustecer o debilitar la cohesión en la sociedad. La sociedad moderna, como sabemos, se constituye en torno al trabajo y éste se ha transformado en un espacio privilegiado para acceder al bienestar e integrarse a la ciudanía social y desarrollar vínculos de solidaridad y cooperación con otros de una misma y/o diversa condición social.

9 Asimismo, en un contexto en el que se han producido fuertes transformaciones en el mundo del trabajo y en el que se cuestiona su validez para generar vínculos cohesivos, se ha podido encontrar formas limitadas en las cuales el trabajo sigue siendo un escenario válido para la cohesión social.

10 La forma en la que se generan los vínculos en el trabajo son la sociabilidad y la asociatividad. La sociabilidad es parte importante de las relaciones que se dan en el mundo del trabajo, ya que éste atributo sigue presente a pesar de la racionalización que ha sufrido este espacio en la sociedad moderna. La sociabilidad que se da en el mundo del trabajo puede ir desde la simple conversación cotidiana hasta la generación de vínculos de afectividad entre compañeros de trabajo (Kim D. O., 2009 ; McGuire G., 2007; Babson S., 2000). Asimismo, estos vínculos de sociabilidad permiten que el mundo del trabajo se convierta en un espacio agradable, que genera mayor bienestar y compromiso con la tarea, en la medida que se logran forjar relaciones entre iguales, existe un reconocimiento a la labor de las personas y las personas se sienten apreciadas en su labor (Hochschild, 1997). Ahora bien, para propiciar las relaciones de sociabilidad, es central poder compartir un espacio de trabajo que permita generar experiencias comunes y relaciones de co-presencialidad ya que esto permite identificarse como parte de un todo común, tener un lenguaje común y vivir experiencias en conjunto (Hodson R., 2004).

11 Por su parte, los vínculos de asociatividad han sido el fundamento de la organización sindical. Tradicionalmente los vínculos en el trabajo se comprenden como vínculos de solidaridad entre iguales en los que prima una identificación común en base a una experiencia de trabajo compartida. Sin embargo, en el marco de una sociedad de mercado fuertemente impactada por nuevos fenómenos de globalización, cabe preguntarse hasta dónde el trabajo mantiene esta fuerza asociativa y hasta dónde desarrolla vínculos de cooperación con otros de condición jerárquica y social diferente. Bien puede ser que el contacto social en el trabajo tenga un carácter conflictivo que refuerza una experiencia y valoración negativa del otro.

12 Como bien señala Rainer Zoll (1998) el desafío de la sociedad actual - más allá de las formas de solidaridad mecánica que suponen empatía y disposición a cooperar entre iguales - es la aceptación y disposición a colaborar con un otro diferente. En este sentido cabe preguntarse hasta dónde el trabajo se constituye como un espacio que potencia estos vínculos o más bien los diluye en el marco de un proceso acelerado de 
individualización.

En suma, la discusión anterior pone de relieve la importancia de interrogarse por el rol que juega el trabajo en el ámbito urbano como espacio de encuentro y valoración social dentro de la nueva sociedad de mercado y hasta dónde él contribuye a debilitar o reforzar los vínculos de cohesión con un otro diferente.

\section{Antecedentes teóricos}

\section{Sociedad de mercado y trabajo}

13 La sociedad chilena ha sufrido un cambio significativo en las cuatro últimas décadas. Los cambios en el modelo de acumulación de orientación neo liberal - que se inicia en 1973 y que con algunas modificaciones se mantiene vigente hasta hoy - han ido transformando la sociedad chilena en una sociedad de mercado, es decir, una sociedad en la cual el mercado no sólo gana centralidad como una forma de intercambio y asignación de recursos económicos, sino como agencia de coordinación, integración y valoración social (Slater D., Tonkiss F., 2001).

En este sentido, el despliegue de la sociedad de mercado no sólo es portador de una nueva experiencia de contacto y vínculo con otros, sino de nuevas formas de valoración social que afectan la cohesión social.

Mirado desde la experiencia de vínculo social con otros, la centralidad del mercado genera una mercantilización y monetarización de las relaciones sociales, una creciente diferenciación de las oportunidades de trabajo y bienestar social, y una segmentación en el acceso a estas últimas conforme a la capacidad de pago de cada cual, lo que tiende a debilitar el contacto entre actores de diferente condición social.

14 En efecto, los datos de la encuesta Eco-social (2007) muestran que 2 de cada 3 chilenos, independiente de su clase social, está de acuerdo con la idea de que es el individuo quien debe buscar sus oportunidades de bienestar. Por su parte, la encuesta sobre Trabajo y Equidad (2008) muestra que el grueso de la población - especialmente los jóvenes - cree que son las características personales como la iniciativa, el talento personal, la responsabilidad y el trabajo duro los elementos que más inciden en el futuro de una persona. Asimismo un $45 \%$ de los entrevistados cree que debería haber mayores premios al esfuerzo individual y un $58 \%$ estima que es tarea de cada uno buscarse oportunidades ${ }^{2}$. Por último, la encuesta Bicentenario (2006-2008), muestra que el $65 \%$ de los entrevistados está de acuerdo con que el trabajo, el esfuerzo y la iniciativa personal son los principales recursos para mejorar la condición de vida de cada cual. Adicionalmente, un $60 \%$ piensa que el éxito económico depende del esfuerzo personal y que la remuneración debe atarse a la productividad de cada cual ${ }^{3}$.

15 Llama la atención, sin embargo, que en la encuesta Trabajo y equidad al testear las preferencias entre la opción de más igualdad o más crecimiento, hay una clara preferencia por una mayor igualdad. Un $65 \%$ opta por más igualdad incluso a costa de un menor crecimiento lo cual puede ser un indicador de un sentimiento y valoración de una mayor solidaridad social. En los último años la sociedad de mercado se ha visto tensionada por diversos movimientos sociales y de los trabajadores (por ejemplo, el movimiento de subcontratistas de CODELCO en 2007) que tienen como fundamento una reivindicación por más y mejores oportunidades de vida para todos y que se traduce en una aspiración solidaria por una ciudadanía más completa que incluya más 
participación e igualación de las oportunidades de vida para el conjunto de la sociedad. En este sentido, la desigualdad en el acceso a las oportunidades de vida generadas por el mercado - y en particular por el mercado de trabajo - está produciendo una reacción a nivel de la sociedad por una mayor igualación de esas mismas oportunidades. En otras palabras, el funcionamiento de la sociedad de mercado está siendo desafiado por el incumplimiento de una de sus promesas fundamentales : la expansión de la igualdad de las oportunidades en las que el trabajo ocupa un lugar central.

\section{Trabajo y cohesión social}

En primer lugar, es importante recalcar que, contrariamente a lo presupuestado por las teorías que afirman una pérdida de centralidad del trabajo, éste ámbito sigue siendo central para una gran parte de la población. (Dahlin E., Kelly E., Moen P., 2008 ; Díaz X., Godoy L., Stecher A., 2005).

La centralidad que posee el trabajo se da en términos de conformación de la rutina cotidiana, identidad y formación de vínculos sociales. Luego del espacio de la familia, el trabajo se constituye como el lugar más importante de conformación de vínculos sociales de amistad y comunidad. Esto se grafica en indicadores como las horas trabajadas, la satisfacción que genera el trabajo en la vida, la formación de vínculos sociales y lazos fuertes al interior del mundo del trabajo y el grado de disfrute que proporcionan los compañeros de trabajo (Dahlin E., Kelly E., Moen P., 2008 ; Stecher A., 2012).

Además, hay que destacar que luego de un siglo de declive en las horas de trabajo estas han tendido a aumentar (Jacobs J. A., Gerson K., 1998) obedeciendo a una necesidad social para poder mantener los niveles de ingreso.

Por otra parte, si bien no reemplaza a la familia -en la medida que ésta se constituye como el principal soporte de relaciones sociales en una sociedad como la chilena- la evidencia da cuenta de que el trabajo sí es más importante que el barrio en la constitución de los vínculos sociales, al menos en el caso de Estados Unidos. (Dahlin E., Kelly E., Moen P., 2008). Como veremos más adelante, una situación similar se comprueba para un caso como el nuestro.

\section{Metodología}

Los resultados que se presentan a continuación provienen de dos fuentes de datos. En primer lugar, se realizó un análisis de las principales tendencias en materia de empleo, utilizando las encuestas periódicas de caracterización socioeconómica, CASEN, aplicadas los años 2000, 2006 y 2011. Se opta por utilizar la serie temporal desde el 2000 para poder observar las últimas tendencias en materia de empleo, considerando que las otras series están disponibles en otras investigaciones ${ }^{4}$. Esta encuesta tiene un carácter bianual y se realiza en hogares a nivel nacional, incluyendo hogares de difícil acceso. Trabaja con una muestra probabilística, geográficamente estratificada y con representatividad a nivel de comunas. Posee un error muestral entre 1 y 4 puntos porcentuales con un $95 \%$ de confianza. Para efectos de este trabajo se utilizaron las comunas pertenecientes a Santiago, Coquimbo, La Serena, Viña del Mar y Valparaíso, y se consideraron solo a trabajadores de zonas urbanas. 
20 Esta decisión obedece a que los análisis sean comparables con los datos de una segunda fuente de información que proviene de la encuesta Anillos de cohesión e integración social, aplicada entre diciembre del 2010 y marzo del 2011 en dichas zonas urbanas. Sin embargo, esa comparación no considera las categorías ocupacionales con las que se trabaja, ya que la caracterización de las formas de cohesión se realiza en base a la clasificación de John Goldthorpe y Robert Erickson, que no es posible de hacer con los datos disponibles de la CASEN.

Esta encuesta aplicó un total de 2564 casos a jefes de hogar y dueñas de casa, y es representativa por nivel socioeconómico. El error muestral asociado a esta encuesta es de 1.96 \%. Para efectos de este análisis se utilizó una sub muestra, sólo de trabajadores, que corresponden a 1414 casos.

\section{Análisis de los resultados}

\section{Trabajo, flexibilidad e integración social}

Para poder comprender la naturaleza de la cohesión social que se funda desde el trabajo, es importante revisar las principales tendencias durante la última década. Esto con el objetivo de identificar si el trabajo posee características que permitan la integración social o si se trata de una experiencia que aboga por la heterogeneidad entre quienes participan en él.

Un primer dato relevante de destacar, es que el trabajo, más que volverse escaso, ha permitido el ingreso de nuevos actores, como son las mujeres y los jóvenes, que han contribuido al crecimiento en la tasa de participación que se fija en torno al 59\% para el año 2013, para el total de la población chilena (Insituto nacional de estadísticas de Chile, 2013).

En el caso de las mujeres de las ciudades mencionadas, la tasa de participación alcanzó al 48,2\% en el año 2011; y en los jóvenes, es interesante destacar la creciente incorporación de los quintiles más bajos quienes sacrifican una mayor instrucción por una incorporación prematura al mercado de trabajo. (Tabla 1). De hecho, en los últimos 10 años, la fuerza laboral ha aumentado su nivel de escolarización pasando de 10,8 a 12,2 años. Sin embargo, este aumento de la escolaridad se da sobre todo en los quintiles superiores quienes alcanzan un nivel promedio de escolarización de 14,88 años. Esto marca una primera segmentación de importancia debido al aumento de la distancia de capital humano entre la fuerza de trabajo calificada y no calificada.

Tabla 1 : Evolución de la tasa de participación urbana de jóvenes de acuerdo a quintil de ingreso autónomo del hogar (2000-2009)

\begin{tabular}{|l|l|l|l|l|l|l|l|l|l|l|l|l|l|l|l|}
\hline \multicolumn{2}{|l|}{2000} & 2006 & & & & & & & & & \\
\hline & I & II & III & IV & V & I & II & III & IV & V & I & II & III & IV & V \\
\hline \hline entre 15 y 19 años & $5,1 \%$ & $4,6 \%$ & $3,1 \%$ & $2,3 \%$ & $1,2 \%$ & $6,2 \%$ & $5,4 \%$ & $4,2 \%$ & $4,0 \%$ & $2,4 \%$ & $4,2 \%$ & $4,2 \%$ & $3,1 \%$ & $2,5 \%$ & $1,4 \%$ \\
\hline \hline 20 a 24 años & $11,7 \%$ & $11,0 \%$ & $11,4 \%$ & $12,0 \%$ & $5,9 \%$ & $10,00 \%$ & $12,2 \%$ & $14,2 \%$ & $12,7 \%$ & $7,3 \%$ & $11,7 \%$ & $13,4 \%$ & $13,8 \%$ & $12,2 \%$ & $6,9 \%$ \\
\hline n & 2053 & 2911 & 3327 & 3606 & 3354 & 1697 & 2768 & 3494 & 4174 & 4318 & 1015 & 1900 & 2339 & 2827 & 3577 \\
\hline
\end{tabular}

FUENTE : ELABORACIÓN PROPIA EN BASE A CASEN 
Adicionalmente, la demanda de trabajo ha sido particularmente importante para los trabajadores más calificados. De hecho, la participación laboral en momentos de crisis económicas ha tendido a disminuir en los quintiles más pobres y a aumentar en los más ricos $^{5}$.

Este aumento en la incorporación al mercado de trabajo ha ido acompañada de una tendencia fluctuante del empleo en el mercado de trabajo. De hecho las tasas de desempleo han oscilado dependiendo de los ciclos macroeconómicos castigando en mayor medida a los quintiles de menores ingresos, que poseen tasas de desempleo que triplican a la de los quintiles de mayor ingreso.

Tabla 2 : Evolución de la tasa de desempleo urbano de acuerdo a quintil de ingreso autónomo del hogar (2000-2011)

\begin{tabular}{|l|l|l|l|}
\hline & 2000 & 2006 & 2011 \\
\hline I & $16,8 \%$ & $10,5 \%$ & $8,3 \%$ \\
\hline II & $8,2 \%$ & $5,9 \%$ & $5,5 \%$ \\
\hline III & $5,4 \%$ & $4,0 \%$ & $3,5 \%$ \\
\hline IV & $3,5 \%$ & $2,9 \%$ & $2,7 \%$ \\
\hline V & $2,1 \%$ & $2,3 \%$ & $1,8 \%$ \\
\hline Total & $6,1 \%$ & $4,5 \%$ & $3,9 \%$ \\
\hline $\mathrm{n}$ & 25937 & 27626 & 20048 \\
\hline
\end{tabular}

Fuente: Elaboración propia en base a CASEN

En términos de condiciones de trabajo, el empleo asumió un carácter eminentemente formal, tendiendo incluso a aumentar en los últimos 10 años desde un 54,6 \% en el año 2000 a un $77 \%$ en el año 2011. No obstante la disminución del empleo informal, aun persiste una importante brecha en términos de quintil de ingreso, ya que la informalidad es más persistente en quintiles de bajos ingresos. 
Tabla 3 : Evolución del empleo según segmentación del mercado de trabajo según quintil de ingreso autónomo del hogar (2000-2011) 6

\begin{tabular}{|c|c|c|c|c|c|c|c|c|}
\hline \multicolumn{3}{|c|}{2000} & \multicolumn{3}{|l|}{2006} & \multicolumn{3}{|l|}{2011} \\
\hline & informal & \begin{tabular}{|l|l} 
formal & $\begin{array}{l}\text { servicio } \\
\text { doméstico }\end{array}$ \\
\end{tabular} & informal & 1 formal & $\begin{array}{l}\text { Servicio } \\
\text { doméstico }\end{array}$ & informal & formal & $\begin{array}{l}\text { servicio } \\
\text { doméstico }\end{array}$ \\
\hline I & $48,9 \%$ & $42,9 \% 8,2 \%$ & $40,0 \%$ & $48,9 \%$ & $11,1 \%$ & $26,0 \%$ & $64,4 \%$ & $9,6 \%$ \\
\hline II & $43,1 \%$ & $49,2 \% 7,7 \%$ & $36,5 \%$ & $53,6 \%$ & $9,9 \%$ & $18,3 \%$ & $72,9 \%$ & $8,8 \%$ \\
\hline III & I $41,1 \%$ & $53,1 \% 5,8 \%$ & $34,7 \%$ & $57,7 \%$ & $7,6 \%$ & $16,5 \%$ & $76,0 \%$ & $67,5 \%$ \\
\hline IV & $41,2 \%$ & $55,0 \% 3,8 \%$ & $34,9 \%$ & $58,6 \%$ & $6,5 \%$ & $20,0 \%$ & $73,4 \%$ & $6,6 \%$ \\
\hline $\mathrm{V}$ & $38,6 \%$ & $60,6 \% 8 \%$ & $36,0 \%$ & $62,8 \%$ & $1,2 \%$ & $14,3 \%$ & $84,0 \%$ & $6,7 \%$ \\
\hline & 5240 & $6894 \quad 697$ & 5112 & 7987 & 857 & 1900 & 8129 & 618 \\
\hline
\end{tabular}

Fuente: Elaboración propia en base a CASEN

El trabajo formal también se ha vuelto más regulado, ya que el uso de contrato de trabajo se ha vuelto cada vez más habitual, incluso entre las microempresas. En el año 2000 , el $77,7 \%$ de los trabajadores dependientes contaba con contrato de trabajo, situación que se incrementa a 85,8 \% el año 2011. Hacia el final del periodo de análisis, se tiende equipara el uso de contrato de trabajo entre hombres y mujeres, así como también tiende a incrementarse el uso de contrato entre trabajadores de todas las edades. También aumenta el uso de contrato en todos los quintiles de ingreso.

Tabla 4 : Situación contractual de los trabajadores dependientes según sexo, edad, quintil de ingreso autónomo del hogar $(2000-2011)^{7}$

\begin{tabular}{|c|c|c|c|c|c|c|}
\hline & \multicolumn{2}{|l|}{2000} & \multicolumn{2}{|l|}{2006} & \multicolumn{2}{|l|}{2011} \\
\hline & $\begin{array}{l}\text { Sí tiene } \\
\text { contrato }\end{array}$ & $\begin{array}{l}\text { No tiene } \\
\text { contrato }\end{array}$ & $\begin{array}{l}\text { Sí tiene } \\
\text { contrato }\end{array}$ & $\begin{array}{l}\text { No tiene } \\
\text { contrato }\end{array}$ & $\begin{array}{l}\text { Sí tiene } \\
\text { contrato }\end{array}$ & $\begin{array}{l}\text { No tiene } \\
\text { contrato }\end{array}$ \\
\hline \multicolumn{7}{|l|}{ Sexo } \\
\hline Hombre & $82,4 \%$ & $17,6 \%$ & $82,1 \%$ & $17,9 \%$ & $88,4 \%$ & $11,6 \%$ \\
\hline Mujer & $76,2 \%$ & $23,8 \%$ & $75,1 \%$ & $24,9 \%$ & $82,5 \%$ & $17,5 \%$ \\
\hline \multicolumn{7}{|l|}{ Edad } \\
\hline $\begin{array}{l}\text { entre } 15 \text { y } 19 \\
\text { años }\end{array}$ & $53,1 \%$ & $46,9 \%$ & $51,2 \%$ & $48,8 \%$ & $64,8 \%$ & $35,2 \%$ \\
\hline 20 a 24 años & $71,9 \%$ & $28,1 \%$ & $73,3 \%$ & $26,7 \%$ & $79,5 \%$ & $20,5 \%$ \\
\hline 25 y más & $81,6 \%$ & $18,4 \%$ & $81,2 \%$ & $18,8 \%$ & $87,2 \%$ & $12,8 \%$ \\
\hline \multicolumn{7}{|l|}{ Quintil } \\
\hline I & $57,7 \%$ & $42,3 \%$ & $67,4 \%$ & $32,6 \%$ & $69,3 \%$ & $30,7 \%$ \\
\hline II & $72,0 \%$ & $28,0 \%$ & $71,3 \%$ & $28,7 \%$ & $79,7 \%$ & $20,3 \%$ \\
\hline
\end{tabular}




\begin{tabular}{|l|l|l|l|l|l|l|}
\hline III & $80,1 \%$ & $19,9 \%$ & $78,9 \%$ & $21,1 \%$ & $84,5 \%$ & $15,5 \%$ \\
\hline IV & $83,8 \%$ & $16,2 \%$ & $81,4 \%$ & $18,6 \%$ & $86,8 \%$ & $13,2 \%$ \\
\hline V & $86,7 \%$ & $13,3 \%$ & $84,8 \%$ & $15,2 \%$ & $92,9 \%$ & $7,1 \%$ \\
\hline n & 7600 & 2221 & 9001 & 2367 & 8478 & 2249 \\
\hline
\end{tabular}

FUENTE: ELABORACIÓN PROPIA EN BASE A CASEN

Es importante destacar que el tipo de contrato de trabajo predominante es el contrato indefinido, que representa un $83,3 \%$ el año 2011. Si bien este tipo de contrato predomina en todos los quintiles, es más frecuente en los quintiles de mayor ingreso.

Sin embargo, a pesar del incremento en el uso de contrato de trabajo, la población economicamente activa no siempre accede a empleo protegido. De hecho sólo el 59,9\% cotiza en una Asociación de fondos previsionales (AFP) ${ }^{8}$ contra un $37,8 \%$ que no cotiza. Como era de esperarse esto último es más prevalente en los quintiles de menores ingresos. Asimismo, se observa un aumento de quienes no cotizan en estos grupos más vulnerables. Esto tiene que ver con la mayor informalidad que se concentra en estos segmentos de menores recursos.

Sin embargo, a partir del 2012, uno de los cambios introducidos en la normativa laboral tiene que ver con la obligatoriedad progresiva que tienen los trabajadores independientes de imponerse en el seguro de previsión social, lo que debiese tender a mejorar la protección de estos sectores.

Tabla 5 : Forma de acceso a la previsión social de la PEA según quintil de ingreso autónomo del hogar (2000-2011)

\begin{tabular}{|l|l|l|l|l|l|l|l|l|l|}
\hline \multicolumn{2}{|l|}{2000} & \multicolumn{2}{l}{2006} & \multicolumn{2}{l|}{2011} \\
\hline & $\begin{array}{l}\text { Otro } \\
\text { sistema }\end{array}$ & AFP & no cotiza & Otro sistema & AFP & no cotiza & $\begin{array}{l}\text { Otro } \\
\text { sistema }\end{array}$ & AFP & no cotiza \\
\hline I & $3,0 \%$ & $46,0 \%$ & $51,1 \%$ & $2,0 \%$ & $45,4 \%$ & $52,7 \%$ & $1,9 \%$ & $35,9 \%$ & $62,2 \%$ \\
\hline II & $3,5 \%$ & $56,0 \%$ & $40,5 \%$ & $2,1 \%$ & $54,5 \%$ & $43,4 \%$ & $1,8 \%$ & $51,8 \%$ & $46,4 \%$ \\
\hline III & $4,8 \%$ & $60,2 \%$ & $35,0 \%$ & $3,0 \%$ & $61,4 \%$ & $35,6 \%$ & $2,0 \%$ & $58,8 \%$ & $39,2 \%$ \\
\hline IV & $6,1 \%$ & $63,5 \%$ & $30,4 \%$ & $4,6 \%$ & $61,3 \%$ & $34,1 \%$ & $2,9 \%$ & $63,2 \%$ & $33,9 \%$ \\
\hline V & $5,0 \%$ & $67,9 \%$ & $27,1 \%$ & $4,2 \%$ & $67,4 \%$ & $28,4 \%$ & $2,8 \%$ & $71,2 \%$ & $26,0 \%$ \\
\hline n & 689 & 7858 & 4902 & 557 & 9601 & 5845 & 158 & 7481 & 1959 \\
\hline
\end{tabular}

Fuente: Elaboración propia en base a CASEN

27 Junto a ello y, dependiendo del quintil de ingreso, entre un $30 \%$ y un $45 \%$ de los trabajadores tienden a mantenerse por 6 años o más en sus empleos. En los quintiles más bajos la inestabilidad es mayor. Sin embargo estos datos no nos permiten saber si 
esa mayor inestabilidad está o no asociada a un cambio de empresa. El caso paradigmático de esta situación son los trabajadores de la construcción que se mueven de una obra a otra, sin necesariamente cambiar de empresa.

Tabla 6 : Estabilidad en el empleo según quintil de ingreso autónomo del hogar (2000-2011)

\begin{tabular}{|c|c|c|c|c|c|c|c|c|c|c|c|c|}
\hline & \multicolumn{4}{|l|}{2000} & \multicolumn{4}{|c|}{2006} & \multicolumn{4}{|l|}{2011} \\
\hline & \begin{tabular}{|l|} 
menos \\
de un \\
año
\end{tabular} & $\mid \begin{array}{l}1 \text { a } 3 \\
\text { años }\end{array}$ & $\begin{array}{l}4 \text { a } 5 \\
\text { años }\end{array}$ & $\begin{array}{l}6 \text { af̃os } \\
\text { o más }\end{array}$ & $\begin{array}{l}\text { menos } \\
\text { de un } \\
\text { año }\end{array}$ & $\mid \begin{array}{l}1 \text { a } 3 \\
\text { años }\end{array}$ & $\begin{array}{l}4 \text { a } 5 \\
\text { años }\end{array}$ & $\begin{array}{l}6 \text { años } \\
\text { o más }\end{array}$ & $\begin{array}{l}\text { menos } \\
\text { de un } \\
\text { año }\end{array}$ & $\begin{array}{l}1 \text { a } 3 \\
\text { años }\end{array}$ & $\begin{array}{l}4 \text { a } 5 \\
\text { años }\end{array}$ & 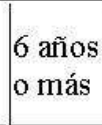 \\
\hline I & $\begin{array}{l}40,5 \\
\% \\
\end{array}$ & $\begin{array}{l}27,3 \\
\% \\
\end{array}$ & $8,0 \%$ & $\begin{array}{l}24,10 \\
\%\end{array}$ & $\begin{array}{l}36,9 \\
\% \\
\end{array}$ & $\begin{array}{l}24,7 \\
\%\end{array}$ & $8,8 \%$ & $\begin{array}{l}29,6 \\
\% \\
\end{array}$ & $\begin{array}{l}37,9 \\
\% \\
\end{array}$ & $\begin{array}{l}28,7 \\
\% \\
\end{array}$ & $8,4 \%$ & $\begin{array}{l}25,0 \\
\%\end{array}$ \\
\hline II & $\begin{array}{l}28,1 \\
\% \\
\end{array}$ & $\begin{array}{l}28,9 \\
\% \\
\end{array}$ & $\begin{array}{l}11,7 \\
\%\end{array}$ & $33.2 \%$ & $\begin{array}{l}30,9 \\
\% \\
\end{array}$ & $\begin{array}{l}26,4 \\
\%\end{array}$ & $8,3 \%$ & $\begin{array}{l}34,5 \\
\% \\
\end{array}$ & $\begin{array}{l}30,9 \\
\%\end{array}$ & $\begin{array}{l}32,1 \\
\% \\
\end{array}$ & $9,0 \%$ & $\begin{array}{l}28,0 \\
\%\end{array}$ \\
\hline III & $\begin{array}{l}23,5 \\
\% \\
\end{array}$ & $\begin{array}{l}30,4 \\
\% \\
\end{array}$ & $\begin{array}{l}12,8 \\
\%\end{array}$ & $33,3 \%$ & $\begin{array}{l}28,6 \\
\%\end{array}$ & $\begin{array}{l}26,6 \\
\%\end{array}$ & $\begin{array}{l}10,1 \\
\%\end{array}$ & $\begin{array}{l}34,7 \\
\%\end{array}$ & $\begin{array}{l}29,0 \\
\%\end{array}$ & $\begin{array}{l}32,1 \\
\% \\
\end{array}$ & $8,3 \%$ & $\begin{array}{l}30,6 \\
\%\end{array}$ \\
\hline $\begin{array}{l}\mathrm{I} \\
\mathrm{V} \\
\end{array}$ & $\begin{array}{l}18,1 \\
\% \\
\end{array}$ & $\begin{array}{l}27,6 \\
\% \\
\end{array}$ & $\begin{array}{l}12,5 \\
\% \\
\end{array}$ & $51,7 \%$ & $\begin{array}{l}22,6 \\
\%\end{array}$ & $\begin{array}{l}27,7 \\
\%\end{array}$ & $9,6 \%$ & $\begin{array}{l}40,2 \\
\%\end{array}$ & $\begin{array}{l}23,4 \\
\%\end{array}$ & $\begin{array}{l}31,8 \\
\%\end{array}$ & $9,4 \%$ & $\begin{array}{l}35,4 \\
\% \\
\end{array}$ \\
\hline V & $\begin{array}{l}13,7 \\
\%\end{array}$ & $\begin{array}{l}26,5 \\
\%\end{array}$ & $\begin{array}{l}11,8 \\
\%\end{array}$ & $48,0 \%$ & $\begin{array}{l}16,4 \\
\%\end{array}$ & $\begin{array}{l}25,9 \\
\%\end{array}$ & $\begin{array}{l}10,1 \\
\%\end{array}$ & $\begin{array}{l}47,7 \\
\%\end{array}$ & $\begin{array}{l}16,1 \\
\%\end{array}$ & $\begin{array}{l}28,6 \\
\%\end{array}$ & $\begin{array}{l}10,2 \\
\%\end{array}$ & $\begin{array}{l}45,2 \\
\%\end{array}$ \\
\hline n & 3008 & 3775 & 1578 & 2206 & 3668 & 3956 & 1427 & 2536 & 2499 & 3138 & 952 & 3640 \\
\hline
\end{tabular}

\section{Fuente: Elaboración propia en base a CASEN}

El trabajo también ha mantenido su perfil en términos de la flexibilidad horaria. Si bien el empleo a tiempo parcial aumentó de un 13,5\% en el año 2000 a un 18,6 \% el año 2011, el trabajo en semana normal es lo que tiende a predominar en el 59,8\% de los trabajadores. Con la entrada en vigencia de la ley de 45 horas semanales, la semana normal de trabajo ha tendido a incrementarse no obstante el 21,6\% de las personas que trabajan aún mantienen una semana de trabajo superior a lo legal. Principalmente quienes se mantienen en esta situación son hombres $(25,4 \%)$, de 25 o más años $(22,7 \%)$ y del quinto quintil de ingreso $(24,8 \%)$.

Finalmente, en términos de remuneraciones el trabajo tampoco ha tendido a aumentar los ingresos de manera considerable sobre el salario mínimo que sí ha aumentado sobre la inflación. Por esta razón, la mayoría de los ingresos generados por el empleo se mantienen alrededor de 1 o 2 salarios mínimos. También es posible apreciar que la brecha de género se mantiene en la composición de los salarios. 
Tabla 7 : Evolución de los salarios, de acuerdo a género y quintil de ingreso autónomo del hogar $(2000-2012)^{9}$

\begin{tabular}{|c|c|c|c|c|c|c|c|c|c|}
\hline & \multicolumn{3}{|l|}{2000} & \multicolumn{3}{|l|}{2006} & \multicolumn{3}{|l|}{2011} \\
\hline & $\begin{array}{l}<\text { de } 1 \\
\text { salario } \\
\text { mínimo }\end{array}$ & \begin{tabular}{|l} 
Entre 1 y \\
2 \\
salarios \\
mínimos
\end{tabular} & $\begin{array}{l}>\text { de } 2 \\
\text { salarios } \\
\text { mínimos }\end{array}$ & $\begin{array}{l}<1 \\
\text { salario } \\
\text { mínimo }\end{array}$ & \begin{tabular}{|l|} 
Entre 1 y \\
2 \\
salarios \\
mínimos
\end{tabular} & $\begin{array}{l}>2 \\
\text { salarios } \\
\text { mínimos }\end{array}$ & $\begin{array}{l}<\text { de } 1 \\
\text { salario } \\
\text { mínimo }\end{array}$ & \begin{tabular}{|l|} 
Entre 1 y \\
2 \\
salarios \\
mínimos
\end{tabular} & $\begin{array}{l}>\text { de } 2 \\
\text { salarios } \\
\text { mínimos }\end{array}$ \\
\hline \multicolumn{10}{|l|}{ Género } \\
\hline Hombre & $9,8 \%$ & $31,9 \%$ & $58,3 \%$ & $12,3 \%$ & $37,6 \%$ & $50,1 \%$ & $8,4 \%$ & $42,8 \%$ & $48,8 \%$ \\
\hline Mujer & $18,9 \%$ & $36,0 \%$ & $45,1 \%$ & $25,2 \%$ & $37,5 \%$ & $37,3 \%$ & $20,9 \%$ & $42,6 \%$ & $36,5 \%$ \\
\hline \multicolumn{10}{|l|}{ Quintil } \\
\hline I & $48,1 \%$ & $48,8 \%$ & $3,0 \%$ & $51,9 \%$ & $46,9 \%$ & $1,2 \%$ & $48,2 \%$ & $50,5 \%$ & $1,3 \%$ \\
\hline II & $24,1 \%$ & $56,1 \%$ & $19,8 \%$ & $33,0 \%$ & $55,1 \%$ & $11,9 \%$ & $25,0 \%$ & $64,6 \%$ & $10,4 \%$ \\
\hline III & $17,2 \%$ & $45,5 \%$ & $37,3 \%$ & $22,4 \%$ & $54,3 \%$ & $23,3 \%$ & $17,5 \%$ & $62,0 \%$ & $20,4 \%$ \\
\hline IV & $6,8 \%$ & $34,6 \%$ & $58,6 \%$ & $11,4 \%$ & $41,2 \%$ & $47,3 \%$ & $8,8 \%$ & $49,2 \%$ & $42,0 \%$ \\
\hline V & $2,1 \%$ & $8,4 \%$ & $89,5 \%$ & $4,2 \%$ & $12,6 \%$ & $83,2 \%$ & $3,0 \%$ & $12,8 \%$ & $84,2 \%$ \\
\hline & 2036 & 5081 & 6185 & 2659 & 6026 & 6269 & 1513 & 4603 & 4679 \\
\hline
\end{tabular}

Fuente: Elaboración propia en base a CASEN

A partir de estos antecedentes es posible observar que, durante la última década, la evolución del mercado de trabajo urbano ha reproducido formas de segmentación relativamente clásicas entre trabajadores formales e informales, con diferentes grado de calificación, lo que genera condiciones de integración al ingreso y bienestar social muy diferentes para los trabajadores pertenecientes a los hogares de los quintiles inferiores y superiores. Para los primeros, el trabajo se presenta como una experiencia fragmentada y precaria; en tanto que, para un segmento de trabajadores pertenecientes a hogares de ingresos medios y altos (quintiles 3 al 5), el trabajo sigue siendo un ámbito relativamente estable, protegido y con una semana de trabajo normal de 45 horas o más. Lo interesante es que, tanto para los unos como para los otros, el trabajo se mantiene como un ámbito central de convivencia social lo que permite entenderlo como un espacio de encuentro y vínculo con otros y, en esta medida, como un ámbito importante de cohesión social. Esto último emerge con fuerza de los datos de nuestra encuesta sobre cohesión social realizada en cinco ciudades chilenas.

\section{Trabajo y cohesión social}

Los datos de esta encuesta señalan la importancia que posee el trabajo como fundamento de integración y convivencia social. El 60,4\% de los trabajadores considera que el trabajo es la actividad más importante que tiene para estar con otros e integrarse a la sociedad, independiente de la categoría ocupacional. De esta forma, el trabajo sigue siendo un eje fundamental para lograr la integración a la sociedad, no sólo desde lo material, sino también porque permite compartir con otros diferentes.

Cabe destacar que en este análisis hemos utilizado la clasificación ocupacional propuesta por Robert Erikson y John Goldthorpe (1993) que permite clasificar a los trabajadores según su nivel de_calificación, relación contractual y tipo de actividad. En la medida que nuestro análisis sólo refiere a los trabajadores urbanos y nuestro « $\mathrm{n}$ » 
muestral considera un número más reducido de entrevistados - aquellos que al momento de la entrevista se encontraban trabajando - hemos reducidos las categorías, por razones de significación, a sólo seis.

Según los datos presentados en el gráfico 1, el trabajo también emerge como un espacio fundamental para generar vínculos con otros. En efecto, si bien el trabajo no parece haber mantenido su relevancia en términos de vínculos de asociatividad - en la medida que sólo un $11.9 \%$ de los entrevistados declara estar afiliado a una asociación gremial o sindical - sí lo es en términos de sociabilidad : el trabajo permite compartir un espacio común independiente del cargo que se ostenta ; la relación de trabajo se extiende más allá del lugar de trabajo y el trabajo permite hacerse amigo de los compañeros.

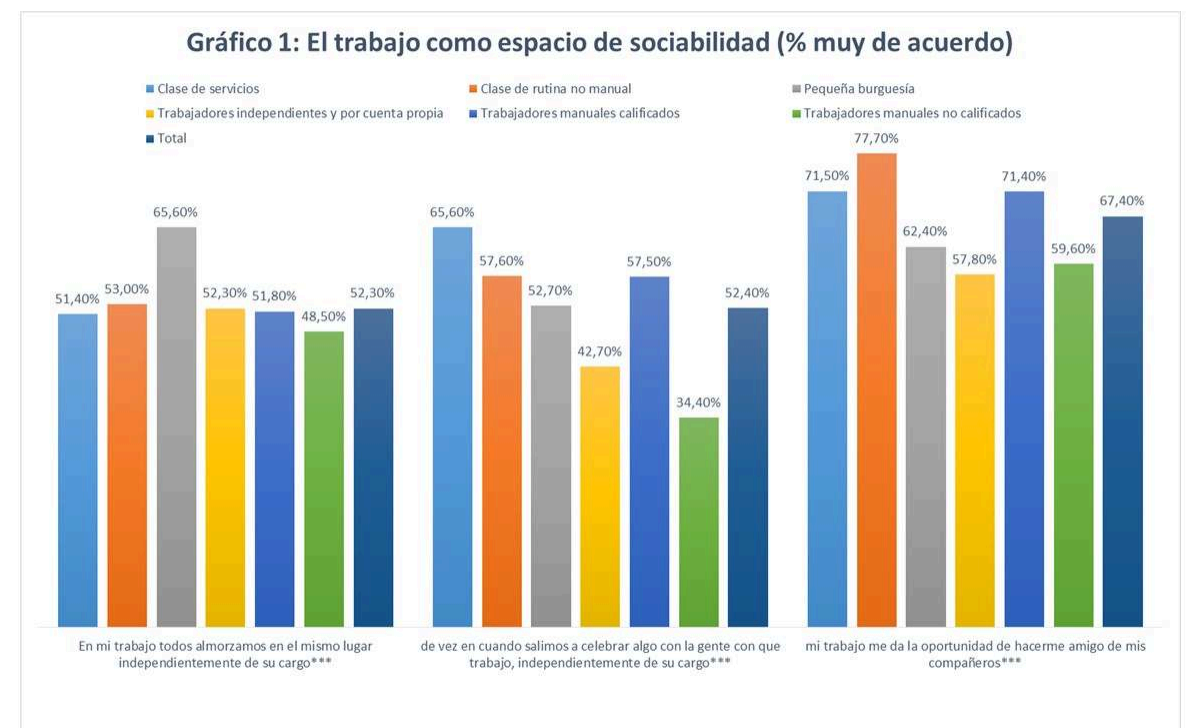

Fuente : Elaboración propia en base a encuesta Proyecto anillo : cultura de integración y cohesión social en las ciudades chilenas ${ }^{10}$

Sin embargo, la sociabilidad no se da de la misma forma en todas las categorías ocupacionales, sino que tiende a ser mayor entre la clase de servicios y los trabajadores cualificados que son, muy probablemente, los que comparten - como veíamos en la sección anterior - condiciones de trabajo más estables, sujetas a un contrato de trabajo y a relaciones de subordinación y dependencia en un lugar de trabajo.

En términos de sociabilidad, el trabajo es un lugar importante para hacerse amigos o conocidos. De hecho, el 82,9\% declara tener conocidos en el trabajo, mientras que el $64,3 \%$ declara tener amigos. Nuevamente, la clase de servicios, la clase de rutina no manual y los trabajadores manuales cualificados son quienes declaran en mayor proporción tener amigos en el trabajo, con un 78,8 \%, 69,1\% y 68,3\% respectivamente. Asimismo, este tipo de trabajadores declara que el trabajo es el lugar donde tiene más amigos ( $41,2 \% ; 42,9 \%$ y $38,8 \%$ respectivamente) mientras que en las otras categorías ocupacionales es el barrio el espacio que se constituye en el núcleo de la amistad.

La posibilidad de constituir al trabajo como espacio de sociabilidad y de generar vínculos de amistad está dada por la naturaleza del otro con el que se establece el encuentro en el lugar de trabajo. En este sentido, el otro que emerge en el lugar de trabajo es distinto de acuerdo a la categoría ocupacional. 


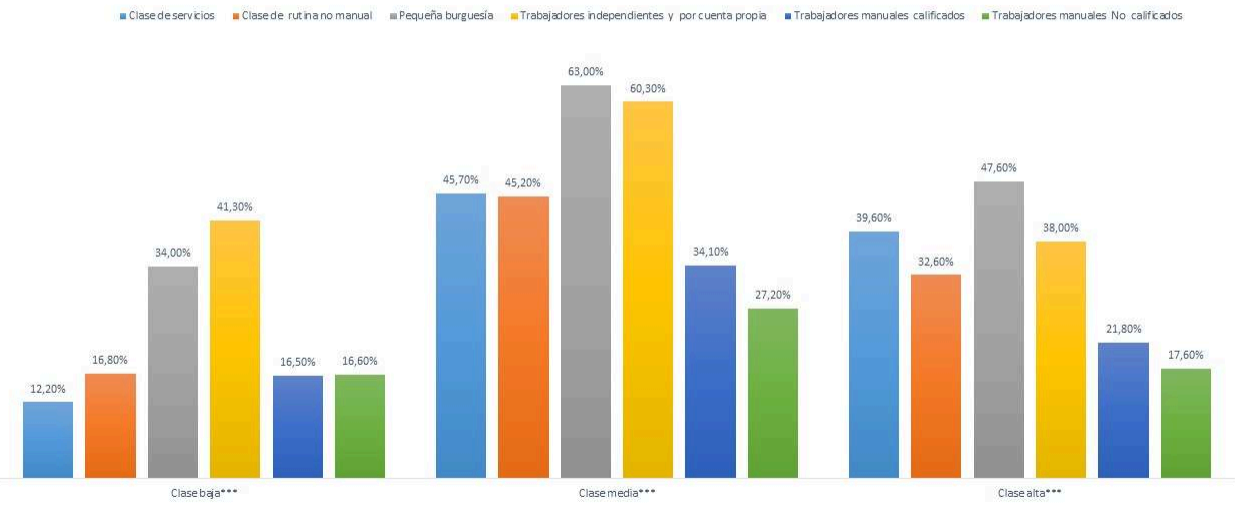

Fuente : Elaboración propia en base a encuesta Proyecto anillo : cultura de integración y cohesión social en las ciudades chilenas ${ }^{11}$ trabajadores, el otro con el que me encuentro en el trabajo y establezco un vínculo de sociabilidad es alguien mayoritariamente de mi misma clase, lo que se observa en el gráfico 3. 


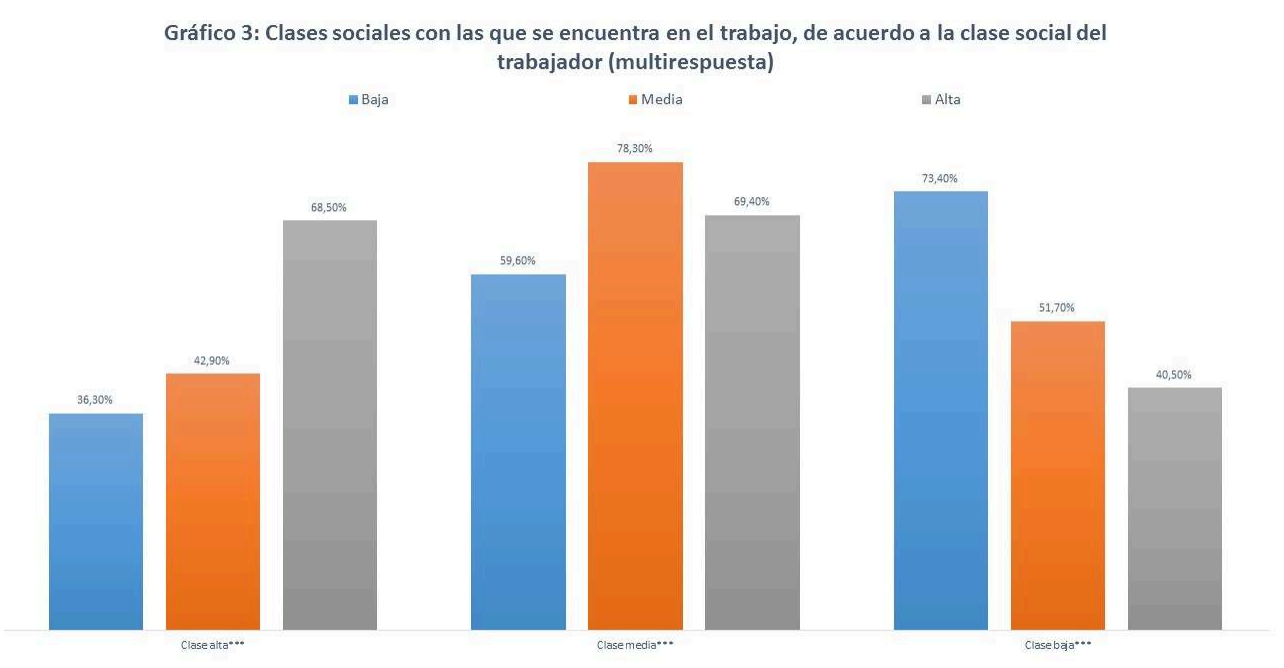

Fuente : Elaboración propia en base a encuesta Proyecto anillo : cultura de integración y cohesión social en las ciudades chilenas ${ }^{12}$. comorías de trabajadores, el trabajo emerge como un valor social fundamental y transversal en todas ellas. El trabajo, no sólo se valora en tanto permite sentirse útil y valioso para la sociedad (86.2\%), sino por el hecho de haberse transformado en un factor fundamental de reconocimiento del otro. Es así como el 94,9\% de nuestros entrevistados señala tener un gran respeto por las personas que se esfuerzan en su trabajo.

41 En suma, en la nueva sociedad de mercado parece haber emergido un proyecto de clase media, basado en el ideal de esfuerzo y movilidad social, valores que están en el núcleo de la transformación de la sociedad chilena y que permite poner al trabajo esforzado como un medio para alcanzar estos ideales. Este aspecto es central en las posibilidades que posee el trabajo para constituirse en un fundamento de cohesión social. Ello en la 
medida que logra sedimentar una valoración común y transversal en todas las categorías ocupacionales. El trabajo y el esfuerzo en el trabajo se transforman en valores ampliamente compartidos y generan un reconocimiento y valoración del otro en tanto alguien que comparte un proyecto de esfuerzo común articulado en torno al trabajo.

\section{Conclusiones}

Uno de los ejes centrales de la transformación de la sociedad chilena ocurrida a lo largo de estas últimas cuatro décadas, ha sido la transformación de sus arreglos institucionales desde formas estado-céntricas hacia estructuras crecientemente mercado-céntricas. Este proceso ha afectado profundamente la realidad y organización del mundo del trabajo y su rol como fundamento de integración y cohesión social.

En este artículo hemos destacado sólo algunas tendencias que afectan la realidad del trabajo en el ámbito urbano y que afectan las formas de integración al bienestar, los vínculos entre los trabajadores y la valoración del trabajo. Mirado desde el punto de vista de la integración y cohesión social de los trabajadores, estas nuevas tendencias propias del desarrollo de esta nueva sociedad de mercado - generan algunas paradojas, tensiones y nuevos desafíos.

Una primera paradoja, es que la valoración del trabajo y del trabajo esforzado como un fundamento de reconocimiento del otro - que es transversal a los diferentes estratos ocupacionales - va acompañada de una fragmentación de la realidad del trabajo que amenaza la integración al bienestar de un porcentaje no despreciable de trabajadores. La economía chilena reproduce un segmento de trabajadores de baja calificación afectados por condiciones de precarización de sus formas de trabajo. Precarización que afecta su integración al bienestar y la generación de vínculos estables con los otros trabajadores; todo lo cual, disminuye la capacidad de integración y sentido de pertenencia social a partir del trabajo. Adicionalmente, estos trabajadores alegan ser discriminados y enfrentar mayores niveles de conflictos en el trabajo. De esta manera, ellos viven una importante disociación entre el rol que juega el trabajo como agencia de integración y su valoración de los otros en tanto trabajadores esforzados, iguales a ellos.

En este sentido un importante desafío para la sociedad, tanto a través del estado como del mercado, es generar condiciones de empleabilidad y protección para estos trabajadores que les permitan reforzar su valoración del trabajo como medio de integración y cohesión social. No hacerlo erosiona uno de los pilares de la sociedad de mercado: su promesa de igualación de las oportunidades sociales a través de la educación y el trabajo.

Un segundo aspecto, aparentemente contradictorio, es que los valores de movilidad y esfuerzo individual ampliamente compartidos van acompañados de importantes vínculos de sociabilidad en el trabajo. Esta contradicción parece resolverse cuando apreciamos que un segmento dominante de trabajadores accede a empleos dependientes y relativamente estables. Esto les permite potenciar sus vínculos con otros trabajadores relativamente similares a ellos llegando a establecer, con algunos, vínculos de amistad que trascienden la esfera del trabajo. De esta manera, no obstante los mayores grados de flexibilidad existentes en el mercado de trabajo, especialmente para los jóvenes y las mujeres, el lugar de trabajo sigue siendo un espacio de encuentro 
para aquellos que logran insertarse mediante un contrato de trabajo, en una jornada completa y por un plazo especificado contractualmente como indefinido. Lo interesante de nuestros datos es que ellos conforman un porcentaje significativo de la fuerza de trabajo urbana más calificada. Para ellos el mundo del trabajo mantiene su centralidad como fundamento cohesivo, si bien con aristas diferentes a las antiguas formas asociativas.

Es interesante observar que esta estabilidad laboral parece ir acompañada de bajos salarios. Esto genera, un trade-off entre remuneración y estabilidad. Al parecer la forma de flexibilidad dominante en el mercado de trabajo urbano chileno tiene que ver con las formas de remuneración más que con las formas de contratación (por ejemplo, a plazo fijo o faena). El precio de la estabilidad son las bajas remuneraciones. Tal vez porque los trabajadores aprecian que con un empleo con contrato más o menos estable pueden acceder al crédito y al endeudamiento que es un patrón ampliamente recurrente entre los trabajadores chilenos. De hecho, de acuerdo a los datos de la última CASEN, el nivel de endeudamiento ${ }^{13}$ de los ocupados alcanza a un $65,1 \%$.

Por último, en este artículo hemos destacado que el trabajo sigue siendo un espacio fundamental para la generación de vínculos y relaciones sociales entre los trabajadores, al tiempo que gana en importancia como un valor de cohesión social. Queda planteado hasta dónde la segmentación del mercado de trabajo que aparece en los datos analizados, se transforma en un estímulo cohesivo entre iguales y, en este sentido, se debilita como espacio de encuentro y cohesión con un otro diferente que, como veíamos con Rainer Zoll (1998), es el desafío cohesivo permanente de las sociedades modernas.

\section{BIBLIOGRAFÍA}

Babson, Steve, « Cross-border trade with Mexico and the prospect for worker solidarity : the case of Mexico », Critical Sociology, vol. 26, n¹-2, 2000, p.13-35.

Dahlin, Eric, Kelly, Erin, Moen, Phyllis, «Is work the new neighborhood ? Social ties in workplace, family and neighborhood». The sociological Quarterly, vol. 49, n²4, 2008, p.719-736.

Díaz, Ximena, Godoy, Lorena, Stecher, Antonio, Significados del Trabajo, Identidad y Ciudadanía. La Experiencia de Hombres y Mujeres en un Mercado Laboral Flexible, Santiago, Cuadernos de Investigación 3, CEM, 2005.

Erikson, Robert, Goldthorpe John, The constant flux,Oxford, Clarendon Press, 1993.

Hochschild, Arlie, «When work becomes home and home becomes work », California Managment Review, vol. 39, n4 1997, p.79-97.

Hodson, Randy, « Work life and social fulfillment: does social affiliation at work reflect a carrot or a stick? », Social Science Quarterly, vol. 85, n², 2004, 221-239.

Jacobs, Jerry A., Gerson, Kathleen, « Who are the overworked americans ? », Review of social economics, vol. 56, n4, 1998, p.442-459. 
Kim, Dong-One, «Employees' perspective on non-union representation : a comparision with unions ", Economic and Industrial Democracy, 2009,p.120-151.

McGuire, Gail, « Intimate at work: a tipology of the social support that workers provide to their network members ", Work and occupations, vol. 34, n², 2007, p.125-147.

Slater, Don, Tonkiss, Fran, Market Society, Polity Press, Reino Unido, 2001.

Stecher, Antonio, « Perfiles identitarios de trabajadores de grandes empresas del retail en Santiago de Chile : aportes psicosociales a la comprensión de las identidades laborales ", Psykhe, vol. 21, $\mathrm{n}^{\circ} 2,2012$, p.9-20

Tironi, Manuel, Tironi, Eugenio, « Ciudad, cohesión y violencia : la experiencia europea y América Latina », in Eugenio Tironi, Redes, Estado y mercado. Soportes de la cohesión social latinoamericana, Santiago de Chile, Uqbar, 2004.

Valenzuela, Eduardo, « Desorganización, solidaridad y movilidad », in Eduardo Valenzuela, Vínculos, creencias e ilusiones. La cohesión social de los latinoamericanos, Santiago de Chile, UqbarEditores, Colección Cieplan, 2008.

Wormald, Guillermo, Ruiz-Tagle, Jaime, « Exclusión Social en el mercado del trabajo : el caso de Chile », Santiago de Chile, Documento de Trabajo N¹06, OIT-Fundación Ford, 1999.

Wormald, Guillermo, Brieba, Daniel, « Institutional change and development in chilean market society », in Alejandro Portes, Lori Smith, Institutions Count, Estados Unidos, University of California Press, 2012.

Zoll, Rainer, « Le défi de la solidarité organique », Sociologie et Sociétés, vol. 30, n² 1998.

\section{NOTAS}

1. La encuesta EcoSocial estudió 7 países de América Latina, incluido Chile y se encuestó a los habitantes de 18 años y más, de ambos sexos, con nacionalidad del país, pertenecientes a todos los niveles socio-económicos de las principales ciudades. En el caso de Chile ellas son: Gran Santiago, Valparaíso, Viña, Antofagasta, Concepción, Talcahuano, Temuco, La Serena y Coquimbo.

2. La encuesta hecha por la Comisión trabajo y equidad (2008) es representativa de la población urbano-rural mayor de 18 años que habita en Chile. Se realizaron 4000 encuestas en hogares, utilizando un muestro aleatorio multietápico. El error muestral fue de $1.5 \%$ a nivel nacional.

3. La encuesta Bicentenario (2009) es realizada por la Universidad católica y Adimark, es representativa de la población urbana mayor de 18 años. Utiliza un instrumento aplicado en hogares y un diseño muestral probabilístico estratificado en cuatro etapas de selección aleatoria. El año 2009 se realizaron 2012 casos, con un error muestral de $2.2 \%$.

4. Ver Wormald y Ruiz Tagle (1999).

5. Durante el periodo analizado se produjeron dos momentos de contracción económica importantes. El primero a partir de la crisis asiática del año 1998 y que se extendió hasta el 2001 ; y el segundo, a partir de la crisis financiera del 2008 que golpeó duramente la economía nacional. 6. La clasificación de segmentación del trabajo urbano se hace considerando los criterios de la Organización internacional del trabajo (OIT) : tamaño de la empresa y categoría ocupacional. Se entiende como empleo informal aquellos que trabajan en microempresas (menos de 5 personas), trabajadores por cuenta propia no profesional o técnico, familiares no remunerados sin profesión y temporeros. El empleo formal se entiende como personas que trabajan en empresas de más de 5 empleados, trabajadores por cuenta propia o familiares no remunerados profesionales y fuerzas 
armadas. El empleo doméstico se analiza como una tercera categoría que podría ser incorporada al sector informal, de acuerdo a la clasificación de la OIT.

7. Se calcula en base a la Casen : «En su trabajo principal, ¿ tiene contrato de trabajo escrito ?».

8. Las AFP son las entidades privadas encargadas por ley de administrar los fondos de jubilación y los seguros de invalidez y accidentes del trabajo de los trabajadores. Todo trabajador dependiente está obligado a imponer un $7 \%$ de su remuneración mensual en una cuenta de capitalización individual en una AFP de su elección.

9. Para el cálculo de los salarios, se consideró el salario mínimo operando ese año.

10. ${ }^{* * *}$ chi cuadrado $<0,01$; ${ }^{* *}$ chi cuadrado $<0,05 ;{ }^{*}$ chi cuadrado $<0,1$

11. ${ }^{* * *}$ chi cuadrado $<0,01 ;{ }^{* *}$ chi cuadrado<0,05; *chi cuadrado $<0,1+$ 'ítems independientes, análisis en base a \% sí para cada una de las clases sociales.

12. ${ }^{* * *}$ chi cuadrado $<0,01 ;{ }^{* *}$ chi cuadrado $<0,05 ; *^{*}$ chi cuadrado $<0,1+$ 'Ítems independientes, análisis en base a \% sí para cada una de las clases sociales.

13. Entendido como personas que han solicitado algún préstamo formal o informal.

\section{RESÚMENES}

En el marco de una sociedad de mercado crecientemente globalizada - como la que se ha venido desarrollando en Chile durante las últimas décadas - los procesos de flexibilización y segmentación de las oportunidades de empleo, así como también los crecientes procesos de individualización de los trabajadores tienden a debilitar la cohesión social entre ellos. Sin embargo, la contracara ha sido un robustecimiento de ella fundada en una revalorización del trabajo como espacio de sociabilidad entre los trabajadores y como un valor social ampliamente compartido. Esta revalorización es relevante en el espacio urbano de una sociedad como la nuestra que se constituye como una sociedad de clase media cohesionada en torno al valor del trabajo y del trabajo duro en particular, así como también de un proyecto compartido de movilidad social fundado en el trabajo. Es el desarrollo combinado de ambos procesos el que genera las tensiones y desafíos que enfrenta el trabajo hoy como fundamento de cohesión social. El presente artículo explora esta relación entre las transformaciones experimentadas en el trabajo y las formas de cohesión posibles de fundar bajo estos contextos. Para ello se realiza el análisis de la encuesta CASEN, que apunta a identificar las características que prevalecen en el trabajo y se analizan datos de trabajadores urbanos provenientes de una encuesta realizada en las ciudades de La Serena, Coquimbo, Valparaíso, Viña del Mar y Santiago en el marco de la encuesta del proyecto de "Integración y Cohesión Social en las Ciudades Chilenas" (Soc-24)*.

* Proyecto en el marco del Programa bicentenario de ciencia y tecnología (PBCT) : Anillos «Cultura de integración y cohesión social en las ciudades chilenas », financiado por la CONICYT.

Dans le contexte d'une société de plus en plus globalisée, telle que la société de marché qui s'est développée au Chili au cours des dernières décennies, les processus de flexibilisation et de segmentation des opportunités d'emploi, ainsi que les processus d'individualisation croissante des travailleurs ont tendance à saper la cohésion sociale. Toutefois, l'autre aspect de ce processus a été un renforcement de celle-ci sur la base d'une nouvelle appréciation du travail comme un espace de sociabilité pour les travailleurs et en tant que valeur sociale largement partagée. Cette revalorisation est particulièrement pertinente dans l'espace urbain, au sein d'une classe 
moyenne où la cohésion repose sur la valeur du travail - et plus particulièrement sur la valeur de travail acharné - ainsi que sur le partage d'un projet commun de mobilité sociale fondée sur le travail. Le développement combiné de ces deux processus génère des tensions et des défis pour l'emploi aujourd'hui, considéré comme le fondement de la " cohésion sociale ».

Cet article explore la relation entre les transformations subies au travail et les formes de cohésion qu'il est possible d'élaborer dans ce contexte. Les auteurs s'appuient sur l'enquête CASEN $^{* *}$, qui vise à identifier les caractéristiques du travail qui prévalent pour les travailleurs urbains. Cette enquête a été menée dans les villes de La Serena, Coquimbo, Valparaiso, Viña del Mar et Santiago du Chili, dans le cadre du projet « Intégration et cohésion sociale dans les villes chiliennes ».

** Encuesta de caracterización Socioeconómica de Chile, aplicada bianualmente por el Ministerio de Desarrollo social.

In the context of an increasingly globalized society - such as the one developing in Chile during recent decades -, the processes of flexibilization and segmentation of employment opportunities, as well as that of growing workers' individualization, tend to undermine social cohesion. At the same time, another consequence of this process has been a new appreciation of "work » as a space enabling workers to socialize, which is increasingly perceived as a shared social value. This positive appropriation of work is especially characteristic of urban spaces, among the middleclass, where cohesion relies upon the value of "work", even "hard work», as well as on the prospect of social mobility thanks to work. Today, these two processes combine to create both tensions and challenges in the labor market and gives rise to the concept of social cohesion.

This article explores the relationship between workplace transformations and the new meaning of social cohesion in this context. To this end, the authors analyze the socio-economic survey CASEN, whose aim is to identify the characteristics that prevail at work and involve urban workers. The study has been conducted in the cities of La Serena, Coquimbo, Valparaiso, Viña del Mar and Santiago de Chile as part of the following global project: «Integration and Social Cohesion in Chilean Cities » (Soc-24).

ÍNDICE

Mots-clés: cohésion, intégration, société de marché, Chili, liens sociaux

Palabras claves: Cohesión, integración, sociedad de mercado, Chile, vínculos sociales

Keywords: Cohesion, integration, market society, Chile, social links

\section{AUTORES}

\section{GUILLERMO WORMALD}

Sociólogo de la Pontificia universidad católica de Chile y Doctor en sociología de la universidad de Sussex, Inglaterra. Trabaja en el Instituto de sociología de la Universidad católica como profesor e investigador. Sus temas de investigación recaen principalmente en el ámbito de la sociología económica, principalmente en las áreas de pobreza y vulnerabilidad social y en sociología del trabajo.

gwormald@uc.cl 


\section{MARIA PAZ TREBILCOCK}

Socióloga, Magíster en sociología y candidato a doctor en sociología de la Pontificia universidad católica de Chile. Académica de la Universidad Alberto Hurtado y del Instituto de sociología.

Investigadora del Centro de desarrollo urbano sustentable (CEDEUS). Sus temas de investigación van en la línea de integración, cohesión social y trabajo.

mtrebilcock@uahurtado.cl 